\title{
Enhancement of Storage Phosphor Plate Images: A C-Language Program
}

\author{
Arch W. Templeton, Louis H. Wetzel, Larry T. Cook, Linda A. Harrison, Donald A. Eckard, \\ William H. Anderson, and Kenneth S. Hensley
}

\begin{abstract}
A C-language software program has been developed for emulating the image enhancement processing of a storage phosphor plate system. This software has been implemented on a VAX 3400 computer. There are 2,100 lines of $\mathrm{C}$-language code in the program. There are seven parameters used to specify the degree of enhancement. The software is being implemented on a single accelerator board.

Copyright $\odot 1992$ by W.B. Saunders Company
\end{abstract}

KEY WORDS: enhancement, C-language program.

7 THE LASER reader of a storage phosphor plate imaging system (Digiscan, Siemens Medical Systems, Iselin, NJ) scans and digitizes the latent image contained in an exposed phosphor storage plate. ${ }^{1}$ The reader has a dedicated microcomputer system for the one-time-only enhancement of each digitized image ( $2 \mathrm{k}$ pixels / row $\times 2 \mathrm{k}$ rows $\times 10$ bits/pixel). The real-time processor automatically applies two enhancement processing algorithms to each digitized image. One algorithm provides for contrast enhancement (gray scale tonal processing). The other emphasizes spatial frequency enhancement (edge processing). The choice of parameters used for a particular image enhancement (Fig 1) are controlled by the type of examination selected. ${ }^{2}$ Parameters may be changed by using the parameter updating system that is part of the phosphor plate system reception terminal. An optical disc stores the original, unenhanced digital data. A gray scale workstation ${ }^{3}$ offers the potential for interactive manipulation of the original digital image. The processed image is printed on a laser film printer.

We have developed a general purpose C-language software package that emulates the dedicated image processing algorithms implemented by the storage phosphor plate microcomputer system.

\section{ENHANCEMENT ALGORITHMS}

The algorithm used for spatial frequency enhancement is unsharp masking. It is implemented by the following equation: (1) QFE = $\mathrm{Q}+\beta\left|\mathrm{Q}-\mathrm{Q}_{\mathrm{us}}\right|$, where $\mathrm{Q}$ is the 10 -bit digitized image, $\beta$ is the degree of enhancement, Qus is the unsharp masked digital image, and QFE is the spatial frequency enhanced digital image. Three parameters specify the degree of spatial frequency enhancement. They are frequency rank (RN), frequency enhancement (RE), and frequency type (RT). The RN can assume one of 10 values. It specifies the peak frequency value $\left(f_{o}\right)$ to be enhanced. The unsharp masked digital image, Qus, is determined by selecting a kernel size, $\mathrm{N}$, and calculating a moving average. Table 1 illustrates the relation between the kernel size $(\mathrm{N})$, the peak of the spatial frequency to be enhanced $\left(f_{o}\right)$, and the sampling rate (SR, pixel $/ \mathrm{mm}$ ).

The RE parameter is selected from a range of 0.1 to 9.9. It is the maximum enhanced value at the frequency, $f_{o}$, selected by $R N$. There are 10 RT-frequency type selectable functions. The identification of the RT functions are $F$, and $P$ through $X$. The function, $\beta$, is calculated as the product of RE and RT.

Following spatial frequency enhancement, the amount of contrast enhancement is determined by the selection of four parameters (Fig 1). These include contrast type (GT, 18 possible lookup tables from " $\mathrm{A}$ " through "O" and $\mathrm{X}, \mathrm{Y}$, $\mathrm{Z}$ ); rotation center ( $\mathrm{GC}$, ranges from 0.3 to 2.6 ); rotation amount (GA, ranges from 0.1 to 4.0 or -0.1 to -4.0$)$; and density shift (GS, ranges from -1.44 to +1.44 ). Figure 2 illustrates the relationship of these four parameters.

\section{PROGRAM}

The organization of the enhancement program is illustrated in Fig 3. The main program contains the tables for detailing RT $(\mathrm{F}, \mathrm{P}$ through $\mathrm{W}$ ) and GT (A through $\mathrm{O}$ ). The GT

From the Department of Diagnostic Radiology, University of Kansas Medical Center, 39th and Rainbow Blvd, Kansas City, KS 66103.

Address reprint requests to Arch $W$. Templeton, $M D$, Department of Diagnostic Radiology, University of Kansas Medical Center, 39th and Rainbow Blvd, Kansas City, KS 66103.

Copyright $\odot 1992$ by W.B. Saunders Company

0897-1889/92/0501-0001\$03.00/0 


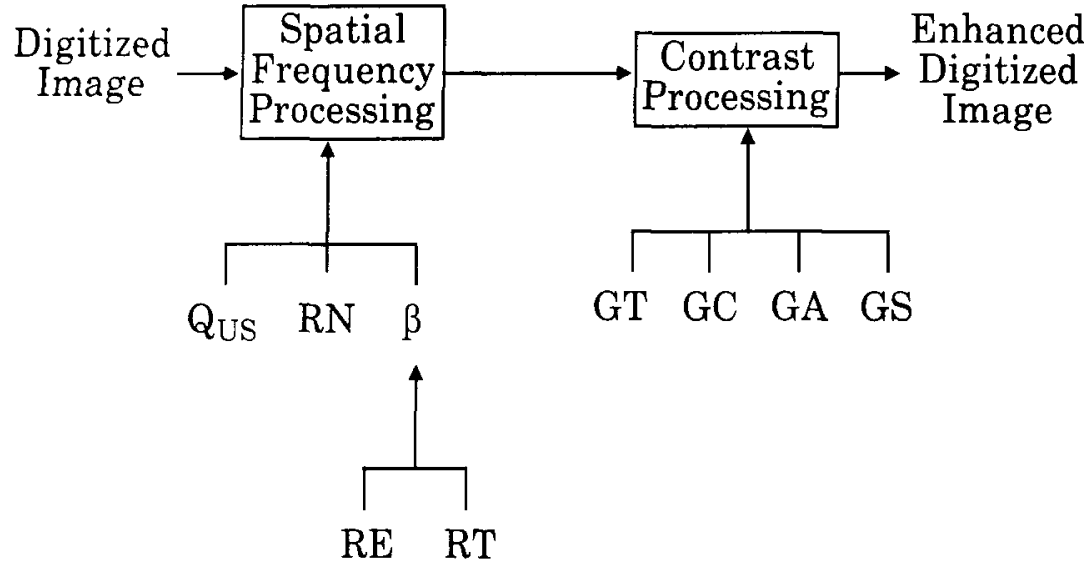

Fig 1. Parameters used for specifying the degree of image enhancement.
Table 1. Kernel Size for Generating Unsharp Mask Image

\begin{tabular}{cccc}
\hline RN & $N(S R=5 \mathrm{pix} / \mathrm{mm})$ & $\mathrm{N}(\mathrm{SR}=6.7 \mathrm{pix} / \mathrm{mm})$ & $\mathrm{N}(\mathrm{SR}=10 \mathrm{pix} / \mathrm{mm})$ \\
\hline 0 & 81 & 109 & 127 \\
1 & 57 & 77 & 115 \\
2 & 41 & 55 & 81 \\
3 & 29 & 39 & 57 \\
4 & 21 & 27 & 41 \\
5 & 15 & 19 & 29 \\
6 & 11 & 13 & 21 \\
7 & 7 & 9 & 15 \\
8 & 5 & 7 & 11 \\
9 & 3 & 5 & 7 \\
\hline
\end{tabular}

NOTE. $N=\left(1.43 / f_{0}\right) \times S R$ where $N$ is the kernel size, $f_{0}$ is the peak of the spatial frequency to be enhanced, and SR is the sampling rate $(\mathrm{pixel} / \mathrm{mm})$. lookup tables map the input digital image data into the desired output gray levels. The RT lookup tables weigh the individual pixel data values for enhancing particular objects in the digital image. The input parameters to the main program are the four contrast values (GA, GT, GC, GS) and the three edge enhancement values (RN, RT, RE). An intermediate contrast curve is generated from the four contrast values (GA, GT, GC, GS) and a $\beta$-table is generated from the edge-enhancement parameter values $(\beta=\mathrm{RE} \times \mathrm{RT})$.

The digital image data is processed by the function titled unsharp mask and final lookup

Fig 2. Lookup table implementation of four parameters selecting the amount of gray-scale enhancement.

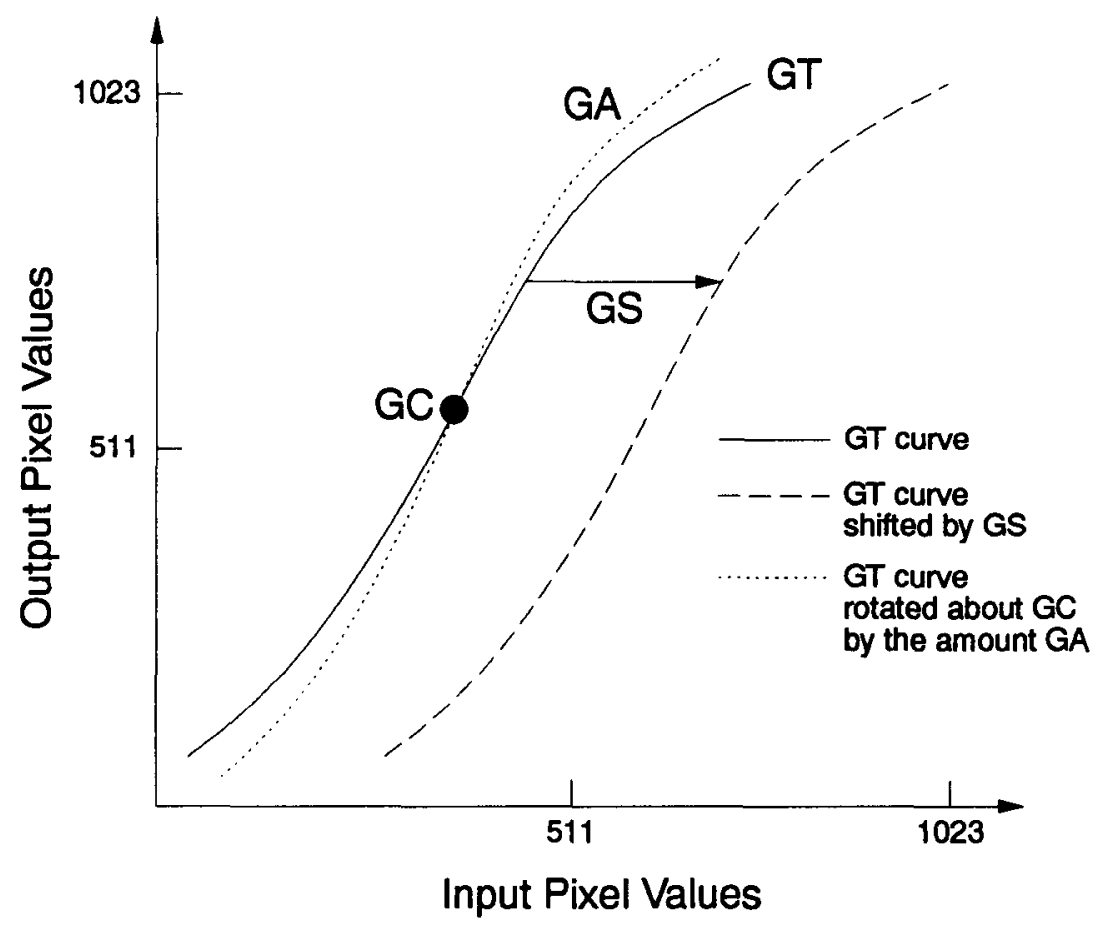




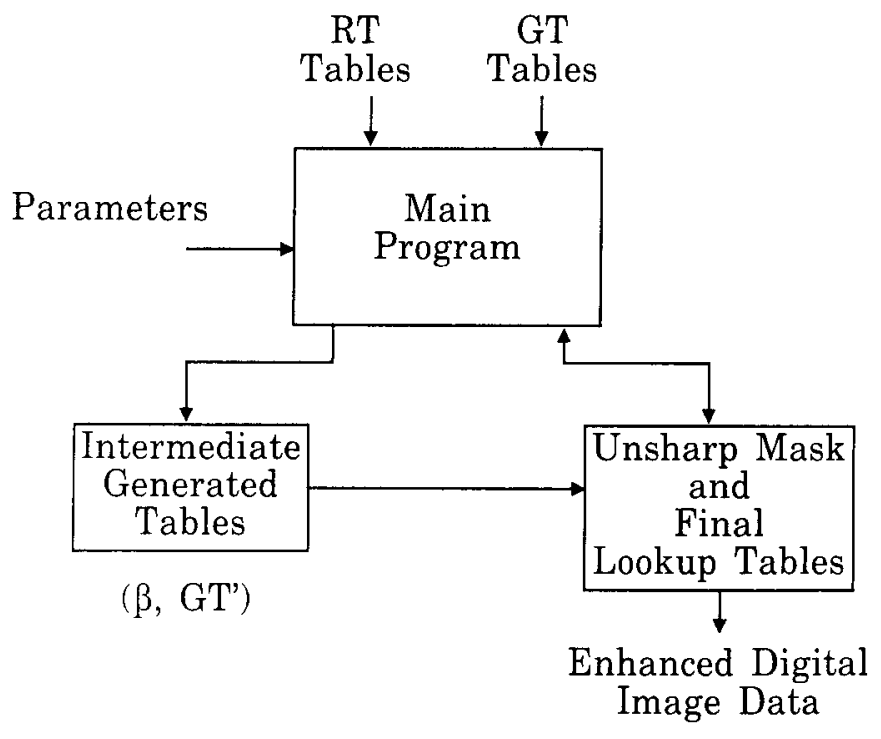

Fig 3. Organization of the enhancement program implemented in C-language software.

FL) Microsoft C-language, version 5.1, using a UNIX V operating system. The total number of code lines is 2,100. The code implementing the unsharp mask and final lookup tables (Fig 3) requires less than 100 lines of C-language software.

We have processed over 120 storage phosphor plate images using the C-language enhancement program. The desired digital image is retrieved from the Digiscan optical disc. The enhancement parameters are selected and entered into the main program. The retrieved
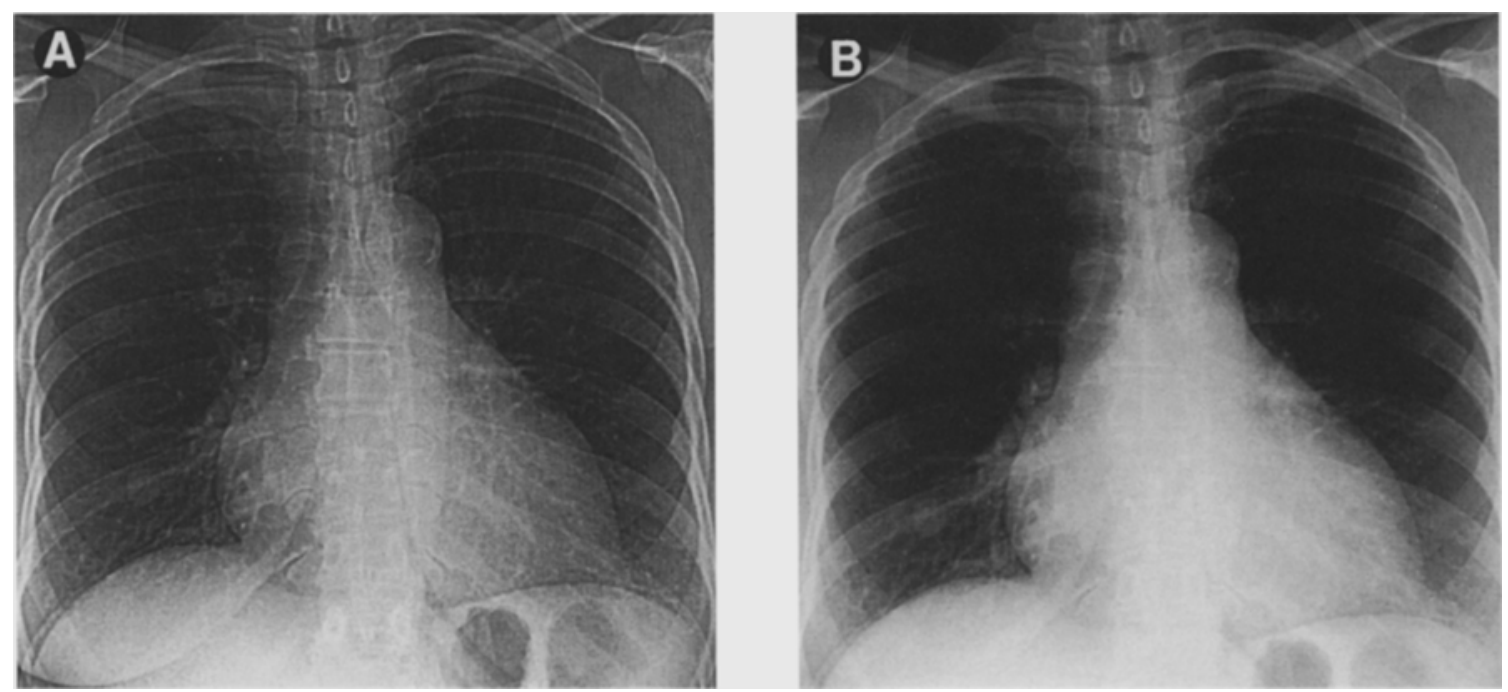

Fig 4. (A) A 49-year-old woman with a malignant nodule in the right lower lobe. Enhancement parameters are: $R \mathbf{N}=4$ (kernel size $=21$ ), $R E=5.0, R T=R, G T=A, G A=0.9, G C=1.5, G S=-0.20$. (B) Enhancement parameters are: $R N=4$ (kernel size $=21$ ), $R E=1.5, R T=R, G T=E, G A=1.0, G C=1.7, G S=-0.20$. 
digital data is transferred to the MicroVAX 3400 for processing by the main program (Fig 3).

The chest image in Fig 4 has been processed using our general purpose $\mathrm{C}$-language enhancement program. Figure $4 \mathrm{~A}$ is a laser-printed image that is identical to the original phosphor plate settings. Figure $4 \mathrm{~B}$ demonstrates a different set of $\mathrm{C}$-language enhancement parameters. Figure $5 \mathrm{~A}$ shows a cervical spine generated by the enhancement $\mathrm{C}$-language program that is identical to the original Digiscan image. Figure $5 \mathrm{~B}$ illustrates the result of using a different set of C-language parameters.

\section{DISCUSSION}

The current real-time processor in the Digiscan laser reader automatically generates and then prints one image. The processor can produce just one enhancement per scanned plate. However, our general purpose C-language software package allows the generation of multiple different enhanced images. The software pack-
Table 2. Processing Times Comparing the VAX 3400 and the AAA Board

\begin{tabular}{ccccc}
\hline Case & Image Size & Kernel Size & VAX Time (sec) & AAA Time (sec) \\
\hline A & $1,760 \times 2,136$ & 41 & 14,823 & 70 \\
A & $1,760 \times 2,136$ & 29 & 7,565 & 53 \\
B & $2,368 \times 1,768$ & 57 & 22,456 & 109 \\
C & $1,760 \times 2,136$ & 21 & 3,457 & 41 \\
\hline
\end{tabular}

age has been incorporated onto a single accelerator board (Adaptive Application Accelerator [AAA], RunTime Technologies, Inc, Irvine, CA). This board uses the IBM PC-AT low cost Industry Standard Architecture bus and the Motorola Digital Signal Processor (DSP) 96002 chip (Austin, TX). The architecture combines a 50 MFLOP Arithmetic Operating Unit, Digital Memory Access (DMA) controllers, and multiple Input/Output $(\mathrm{I} / \mathrm{O})$ ports. Software includes a $\mathrm{C}$-compiler for generating AAA runtime code.

We have obtained preliminary results comparing the times required to modify four phosphor plate images using the AAA board and our standard VAX 3400 (Table 2). The time for
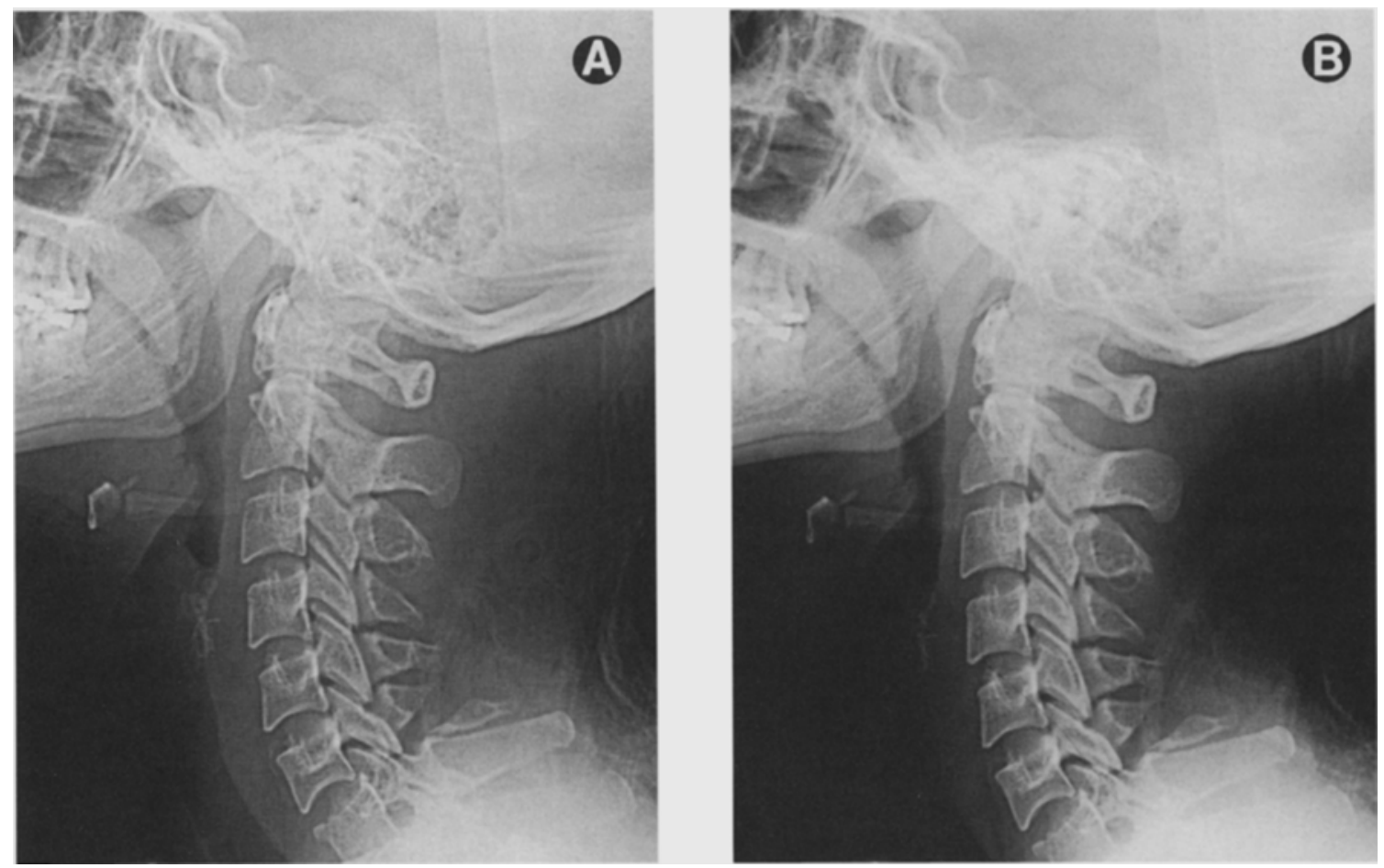

Fig 5. (A) A 31-year-old male with a compression chip fracture involving the body of $\mathrm{C7}$ and an avulsion fracture involving the spinous process of C6. Enhancement parameters are: $R \mathbf{N}=5$ (kemel size $=19$ ), $R E=5.0, R T=P, G T=A, G A=1.0, G C=0.5, G S=$ 0.3. (B) Enhancement parameters are: $R N=5$ (kernel size $=19$ ), $R E=2.5$, RT $=P, G T=E, G A=1.0, G C=0.5, G S=0.4$. 
processing using the VAX is about 100 times greater than the processing time using the AAA board. Table 2 documents these results for several kernels ranging from 21 to 57 . The results indicate that the time required is dependent on the size of the kernel chosen. Improvements in the code may yield more time reductions for the AAA board in the future.

\section{REFERENCES}

1. Blume H: Stimulable phosphor systems: Technical aspects, in Peppler WW, Alter A (eds): Proceedings of Chest Imaging Conference '87. Madison, WI, Medical Physics Publishing, 1987, pp 194-207

2. Murphey MD, Huang HK, Siegel EL, et al: Clinical experience in the use of photostimulable phosphor radiographic systems. Invest Radiol 26,(6):590-597, 1991

3. Murphey MD, Bramble JM, Cox GG, et al: Experience in the use of an image-processing workstation for a photostimulable phosphor and radiographic system. J Digit Imag 3(1):60-65, 1990 